\title{
AN INTERESTING INFINITE SERIES AND ITS IMPLICATIONS TO OPERATOR THEORY
}

\author{
MELANIE HENTHORN-BAKER
}

\begin{abstract}
The following is a discussion regarding a specific class of operators acting on the space of entire functions, denoted $H(\mathbb{C})$. A diagonal operator $D$ on $H(\mathbb{C})$ is defined to be a continuous linear map, sending $H(\mathbb{C})$ into $H(\mathbb{C})$, that has the monomials $z^{n}$ as its eigenvectors and $\left\{\lambda_{n}\right\}$ as the corresponding eigenvalues.

A closed subspace $M$ is invariant for $D$ if $D f \in M$ for all $f \in$ $M$. The study of invariant subspaces is a popular topic in modern operator theory. We observe that the closed linear span of the orbit, which we write $\overline{\operatorname{span}}\left\{D^{k} f: k \geq 0\right\}=\overline{\operatorname{span}}\left\{\sum_{n=0}^{\infty} a_{n} \lambda_{n}^{k} z^{n}: k \geq\right.$ $0\}$, is the smallest closed invariant subspace for $D$ containing $f$. If every invariant subspace for a diagonal operator $D$ on $H(\mathbb{C})$ can be expressed as a closed linear span of some subset of the eigenvectors of $D$, we say that $D$ admits spectral synthesis on $H(\mathbb{C})$. Until recently, it was not known whether or not every diagonal operator on $H(\mathbb{C})$ admitted spectral synthesis.

This article focuses on using techniques from calculus and linear algebra to construct a class of operators which fail spectral synthesis on $H(\mathbb{C})$. If the reader is not familiar with the operator theory definitions provided in the background, he or she can still appreciate the construction of an interesting infinite series relying on properties of logarithms, various convergence tests, and Cramer's Rule.
\end{abstract}

\section{BACKGROUND}

For more than sixty years, operator theorists have been trying to resolve the Invariant Subspace Problem; that is, they have been trying to determine whether or not every operator on a separable, infinite dimensional Hilbert space $\mathcal{H}$ has a nontrivial invariant subspace. (An invariant subspace for an operator $T$ is a closed subspace $M$ of $\mathcal{H}$ satisfying $T x \in M$ for every vector $x$ in $M$.) No one knows who originally posed the question, but it seems that it arose in the late 1940's after Beurling published his most famous paper on invariant subspaces of shift operators (Yadav [21]). In their attempts to resolve this famous open problem, mathematicians have branched out in a variety of ways. One method that has been employed is to examine this question with a specific class of operators which exhibit a particularly 


\section{HENTHORN-BAKER}

nice structure, called diagonal operators. In the setting of a Hilbert space $\mathcal{H}$, a diagonal operator $D$, is defined to be a continuous, linear map such that $D\left(e_{n}\right)=\lambda_{n} e_{n}$ for all $n \geq 0$, where $\left\{e_{n}\right\}_{n=0}^{\infty}$ is an orthonormal basis for $\mathcal{H}$. In order to learn more about diagonal operators on Hilbert spaces, some mathematicians study the analogue of this type of operator on different spaces. Studying the invariant subspaces of diagonal operators will hopefully facilitate the solving of the Invariant Subspace Problem.

A Hilbert space, of course, is a special kind of Banach space. In the more general setting of a Banach space, it has been shown that there do in fact exist bounded linear operators that do not have any nontrivial invariant subspaces. This construction was first done by Per Enflo in 1976, although it was not actually published until 1987. In the meantime, C. J. Read used the same general ideas as Per Enflo and published a similar result in 1984. Read also strengthened Enflo's result and constructed a bounded linear operator on the Banach space $\ell_{1}$ without nontrivial invariant subspaces [21].

The quest for a solution to the invariant subspace problem also gave birth to a related field of study called spectral synthesis. Suppose that $\mathbb{X}$ is a complete, metrizable topological vector space. The most obvious invariant subspace for an operator $D$ acting on $\mathbb{X}$ would be the closed linear span of the orbit of any vector $f$ from $\mathbb{X}$, which we can denote $\overline{\operatorname{span}}\left\{D^{k} f: k \geq 0\right\}$. Now, recalling the definition of a diagonal operator on $\mathcal{H}$, we have that $\overline{\operatorname{span}}\left\{D^{k} f: k \geq 0\right\}$ is actually the closed linear span of some subset of eigenvectors. Clearly, all closed linear spans of subsets of eigenvectors are invariant for $D$, but the interesting question is whether or not these are the only closed invariant subspaces for $D$.

If an operator $T: \mathbb{X} \rightarrow \mathbb{X}$, where $\mathbb{X}$ is any complete, metrizable topological vector space, has the property that all of its invariant subspaces can be expressed as the closed linear span of some set of eigenvectors for $T$, then we say that $T$ admits spectral synthesis on $\mathbb{X}$. In short, we say $T$ is synthetic. If $T$ fails to admit spectral synthesis (in short, $T$ is nonsynthetic), then $T$ has some invariant subspaces that cannot be expressed as the closed linear span of some set of eigenvectors for $T$.

This was a very popular area of exploration in the mid-twentieth century. A theorem summarizing the relevant works coming from a combination of Brown, Shields, and Zeller [1], Nikol'skii [10, 11], Sarason [13, 14], Scroggs [15], Sibilev [18], and Wermer [19] expressing a multitude of conditions equivalent to a diagonal operator failing synthesis on a Hilbert space can be found in a paper by Marin and Seubert [9]. This result demonstrates the complexity of the spectral synthesis question for diagonal operators on Hilbert spaces. We will extract the parts of this extensive theorem most relevant to this article. 


\section{AN INFINITE SERIES APPLIES TO OPERATOR THEORY}

Theorem 1. Let $\mathcal{H}$ be a separable infinite dimensional Hilbert space and let $D$ be any bounded linear operator on $\mathcal{H}$. Suppose there exists an orthonormal basis $\left\{e_{n}\right\}$ for $\mathcal{H}$ and a sequence $\left\{\lambda_{n}\right\}$ of complex numbers for which $D e_{n}=\lambda_{n} e_{n}$ for all $n \geq 0$. Then $\left\{\lambda_{n}\right\}$ is bounded and, if $\lambda_{n} \neq \lambda_{m}$ for all $n \neq m$, the following are equivalent.

(i) D admits spectral synthesis.

(ii) There does not exist a nonzero sequence $\left\{\omega_{n}\right\}$ of complex numbers in $\ell_{1}$ for which $\sum_{n=0}^{\infty} \omega_{n} \lambda_{n}^{k}=0$ for all $k \geq 0$.

As early as the 1920 's, mathematicians were exploring the possibility of getting a series, such as $\sum_{n=0}^{\infty} \omega_{n} \lambda_{n}^{k}$, to be identically zero for all integers $k \geq 0$, even though the terms of the series were not identically zero. Many articles were published under this category of "representing zero". The work done in 1921 by one mathematician by the name of Wolff on this particular topic became very relevant in the field of spectral synthesis upon the publication of Theorem 1 in the early 1990's. Wolff's construction results in the first known example of a diagonal operator failing spectral synthesis on a Hilbert space. This is rather interesting, because Wolff is credited with this groundbreaking example of a nonsynthetic operator, even though the term spectral synthesis did not come about until decades after his construction.

Example 1. (Wolff [20]) Let $\left\{D_{j}\right\}_{j=1}^{\infty}$ be any countable collection of disks, each having a unique center at a point $\lambda_{j}$ in the unit disk and radius $r_{j}<1$, satisfying the property that the planar Lebesgue measure, denoted $m_{2}$, of the set $\mathbb{D} \backslash\left(\bigcup D_{j}\right)$ is zero. Then, $\sum_{j=1}^{\infty} \omega_{j} \lambda_{j}^{m}=0$ for all $m \geq 0$.

Notice that $\left\{\lambda_{n}\right\}$ is bounded, so the map $D$ with eigenvalues $\left\{\lambda_{j}\right\}$ is a well-defined operator acting on $\ell_{2}$. Consider the sequence $\omega_{j}:=r_{j}^{2} \lambda_{j}$ for all $j \geq 1$. Clearly, $\left\{\omega_{j}\right\}$ is not identically zero, and according to Wolff, $\sum_{j=1}^{\infty} \omega_{j} \lambda_{j}^{k} \equiv 0$ for all $k \geq 0$. This construction yielded the first known example of a diagonal operator failing spectral synthesis on a Hilbert space.

The space of functions analytic on the entire complex plane, which we will denote $H(\mathbb{C})$, is a well-studied space, even in graduate level analysis courses. $H(\mathbb{C})$ is a complete metrizable topological vector space. Spectral synthesis of diagonal operators on $H(\mathbb{C})$ have been studied by Deters [3, 4 , 5], Marin [9], Seubert [16], and Wade [17] amongst others. It is worthwhile to discuss some of the existing results concerning this subject.

Any function in $f \in H(\mathbb{C})$ can be expressed as power series $\sum_{n=0}^{\infty} a_{n} z^{n}$ that converges for all $z$ in the plane. Specifically, the radius convergence theorem leads us to the following conclusion.

Theorem 2. (Marin [9]) Suppose $\left\{\lambda_{n}\right\}$ is a sequence of complex numbers. Then, $D\left(\sum_{n=0}^{\infty} a_{n} z^{n}\right)=\sum_{n=0}^{\infty} \lambda_{n} a_{n} z^{n}$ defines a continuous, linear map 


\section{HENTHORN-BAKER}

from $H(\mathbb{C})$ to $H(\mathbb{C})$ if and only if

$$
\limsup \left|\lambda_{n}\right|^{1 / n}<\infty
$$

In regard to spectral synthesis, we have the following theorem to parallel the one existing on Hilbert spaces.

Theorem 3. ([9]) Let $D$ be any diagonal operator on $H(\mathbb{C})$ having distinct eigenvalues $\left\{\lambda_{n}\right\}$. Then the following are equivalent.

(i) D admits spectral synthesis.

(ii) There does not exist a sequence $\left\{\omega_{n}\right\}$ of complex numbers, not identically zero, for which limsup $\left|\omega_{n}\right|^{1 / n}=0$ and $0 \equiv \sum_{n=0}^{\infty} \omega_{n} \lambda_{n}^{k}$ for all $k \geq 0$.

\section{An Infinite Dimensional System of Equations}

As mentioned earlier, Wolff did not know of the term spectral synthesis, and yet he was able to construct a very important operator. Even if unfamiliar with much operator theory, one can dive into the construction of nonsynthetic operators by relying instead on calculus and linear algebra skills. In an introductory linear algebra course, we learn techniques for solving systems of equations. Given a set of distinct, nonzero complex numbers $\left\{\lambda_{n}\right\}_{n=0}^{2}$, consider the system

$$
\begin{gathered}
\beta_{0}^{(3)}+\beta_{1}^{(3)}+\beta_{2}^{(3)}=1 \\
\beta_{0}^{(3)} \lambda_{0}+\beta_{1}^{(3)} \lambda_{1}+\beta_{2}^{(3)} \lambda_{2}=0 \\
\beta_{0}^{(3)} \lambda_{0}^{2}+\beta_{1}^{(3)} \lambda_{1}^{2}+\beta_{2}^{(3)} \lambda_{2}^{2}=0 .
\end{gathered}
$$

We can express this system equivalently as

$$
\sum_{n=0}^{2} \beta_{n}^{(3)} \lambda_{n}^{m}= \begin{cases}1 & \text { if } m=0 \\ 0 & \text { if } m=1,2\end{cases}
$$

or in its matrix form

$$
\left[\begin{array}{ccc}
1 & 1 & 1 \\
\lambda_{0} & \lambda_{1} & \lambda_{2} \\
\lambda_{0}^{2} & \lambda_{1}^{2} & \lambda_{2}^{2}
\end{array}\right]\left[\begin{array}{l}
\beta_{0}^{(3)} \\
\beta_{1}^{(3)} \\
\beta_{2}^{(3)}
\end{array}\right]=\left[\begin{array}{l}
1 \\
0 \\
0
\end{array}\right]
$$

Using Cramer's Rule, we can easily solve for $\left\{\beta_{n}^{(3)}\right\}_{n=0}^{2}$. For example, 
AN INFINITE SERIES APPLIES TO OPERATOR THEORY

$$
\beta_{0}^{(3)}=\frac{\operatorname{det}\left[\begin{array}{ccc}
1 & 1 & 1 \\
0 & \lambda_{1} & \lambda_{2} \\
0 & \lambda_{1}^{2} & \lambda_{2}^{2}
\end{array}\right]}{\operatorname{det}\left[\begin{array}{ccc}
1 & 1 & 1 \\
\lambda_{0} & \lambda_{1} & \lambda_{2} \\
\lambda_{0}^{2} & \lambda_{1}^{2} & \lambda_{2}^{2}
\end{array}\right]} .
$$

Let us take a minute to study the matrix in this denominator. A matrix with this form is called a Vandermonde matrix, and its determinant is well known to be

$$
\prod_{0 \leq i<j \leq 2}\left(\lambda_{j}-\lambda_{i}\right)=\left(\lambda_{2}-\lambda_{1}\right)\left(\lambda_{2}-\lambda_{0}\right)\left(\lambda_{1}-\lambda_{0}\right)
$$

Moving on to the determinant in the numerator, we can expand by the first column and, with some minor adjustments, end up with another Vandermonde matrix.

$$
\begin{aligned}
\operatorname{det}\left[\begin{array}{ccc}
1 & 1 & 1 \\
0 & \lambda_{1} & \lambda_{2} \\
0 & \lambda_{1}^{2} & \lambda_{2}^{2}
\end{array}\right] & =\operatorname{det}\left[\begin{array}{cc}
\lambda_{1} & \lambda_{2} \\
\lambda_{1}^{2} & \lambda_{2}^{2}
\end{array}\right] \\
& =\lambda_{1} \lambda_{2} \operatorname{det}\left[\begin{array}{cc}
1 & 1 \\
\lambda_{1} & \lambda_{2}
\end{array}\right] \\
& =\lambda_{1} \lambda_{2}\left(\lambda_{2}-\lambda_{1}\right) .
\end{aligned}
$$

Then,

$$
\beta_{0}^{(3)}=\left(\frac{\lambda_{1}}{\lambda_{1}-\lambda_{0}}\right)\left(\frac{\lambda_{2}}{\lambda_{2}-\lambda_{0}}\right)
$$

This leads us to the following conclusion.

Lemma 1. Let $\left\{\lambda_{n}\right\}_{n=0}^{N}$ be a set of distinct, nonzero complex numbers. Then for each $N$,

$$
\sum_{n=0}^{N}\left(\prod_{j \neq n}^{N} \frac{\lambda_{j}}{\lambda_{j}-\lambda_{n}}\right) \lambda_{n}^{m}= \begin{cases}1 & \text { if } m=0 \\ 0 & \text { if } 1 \leq m \leq N .\end{cases}
$$

The $(N+1) \mathrm{x}(N+1)$ system of equations in this lemma is easily solved using Cramer's Rule and properties of the Vandermonde matrix. The full proof is available in my dissertation, [7]. The reader may be wondering why we are solving the system with $\left[\begin{array}{lll}1, & 0, & 0\end{array}\right]^{T}$ as the column vector on the right-hand side of the matrix equation, rather than $\left[\begin{array}{lll}0, & 0, & 0\end{array}\right]^{T}$. Please note that if we were to solve the equation with this zero vector,

MISSOURI J. OF MATH. SCI., SPRING 2016 


\section{HENTHORN-BAKER}

we would end up with the trivial solution as the only solution. Since this would certainly not be interesting, we tweaked the system and will make adjustments later in this article via a change of variable.

An interesting question that arises from this result is whether or not we can extend this notion to infinite dimensions. In other words, if $\left\{\lambda_{n}\right\}$ is a sequence of distinct, nonzero complex numbers and if $\beta_{n}=\prod_{j \neq n}^{\infty} \frac{\lambda_{j}}{\lambda_{j}-\lambda_{n}}$, is $\sum_{n=0}^{\infty} \beta_{n} \lambda_{n}^{k}=0$ for all integers $k \geq 1$ ?

To resolve this question, we would have to verify that the $\beta_{n}$ are each in fact well-defined infinite products. Then we would need to verify that the growth rates of the sequences $\left\{\lambda_{n}\right\}$ and $\left\{\beta_{n}\right\}$ are such that the infinite sum $\sum_{n=0}^{\infty} \beta_{n} \lambda_{n}^{k}$ does in fact converge for any given natural number $k$. Finally, we would need to verify if the series in fact converges to zero for all natural numbers $k$.

\section{A Class of Nonsynthetic Diagonal Operators on $H(\mathbb{C})$}

Theorem 4. Suppose that $D$ is a diagonal operator on $H(\mathbb{C})$ having distinct, nonzero eigenvalues $\left\{\lambda_{n}\right\}$ which satisfy $\lim \sup \left|\frac{\lambda_{n}}{\lambda_{n+1}}\right|<1$. Then $D$ fails spectral synthesis on $H(\mathbb{C})$.

Proof. Note that since limsup $\left|\frac{\lambda_{n}}{\lambda_{n}+1}\right|<1$, there exists a real number $q$ with $0<q<1$ and a positive integer $N_{0}$ such that for all $n \geq N_{0}$, we know $\frac{\left|\lambda_{n}\right|}{\left|\lambda_{n+1}\right|}<q$. This means that when $n \geq N_{0}$,

$$
\left|\lambda_{n}\right|<q\left|\lambda_{n+1}\right|<q^{2}\left|\lambda_{n+2}\right|<\cdots .
$$

Consequently, for any pair of positive integers $a$ and $b$, with $a>b \geq N_{0}$,

$$
\left|\lambda_{b}\right|<q^{a-b}\left|\lambda_{a}\right| .
$$

It is clear by this equation that $\left|\lambda_{j}\right|$ are increasing for $j$ larger than $N_{0}$. Now, let $n$ be an arbitrary fixed nonnegative integer and consider

$$
\beta_{n}=\prod_{j \neq n}^{\infty} \frac{\lambda_{j}}{\lambda_{j}-\lambda_{n}}
$$

First, we will see that $\beta_{n}$ is well-defined. Notice that $\lim _{j \rightarrow \infty}\left|\lambda_{j}\right|=\infty$, and consequently, for any fixed $n$,

$$
\lim _{j \rightarrow \infty} \frac{\lambda_{j}}{\lambda_{j}-\lambda_{n}}=1 \quad \text { and } \quad \lim _{j \rightarrow \infty} \frac{\lambda_{n}}{\lambda_{j}-\lambda_{n}}=0 .
$$

This means we can choose a positive integer $N_{1}$ so that for all $j \geq N_{1}$,

$$
\operatorname{Re}\left(\frac{\lambda_{j}}{\lambda_{j}-\lambda_{n}}\right)>0 \text { and } \frac{\left|\lambda_{n}\right|}{\left|\lambda_{j}-\lambda_{n}\right|}<\frac{1}{2} .
$$




\section{AN INFINITE SERIES APPLIES TO OPERATOR THEORY}

Also, let us assume that $N_{1}$ was chosen to be larger than both $N_{0}$ and $n$. Note that showing $\beta_{n}$ is well-defined is equivalent to showing that the "tail" $\prod_{j=N_{1}}^{\infty} \frac{\lambda_{j}}{\lambda_{j}-\lambda_{n}}$ converges. Now, recall the inequality stating when $|z|<\frac{1}{2}$,

$$
\frac{1}{2}|z| \leq|\log (1+z)| \leq \frac{3}{2}|z| .
$$

(This can be shown by examining the power series expansion of $\log (1+z)$. See Conway [2, p 165].) Using this, along with equations 1 and 2,

$$
\begin{aligned}
\sum_{j=N_{1}}^{\infty}\left|\log \left(\frac{\lambda_{j}}{\lambda_{j}-\lambda_{n}}\right)\right| & =\sum_{j=N_{1}}^{\infty}\left|\log \left(1+\frac{\lambda_{n}}{\lambda_{j}-\lambda_{n}}\right)\right| \\
& \leq \frac{3}{2} \sum_{j=N_{1}}^{\infty} \frac{\left|\lambda_{n}\right|}{\left|\lambda_{j}-\lambda_{n}\right|} \\
& \leq \frac{3}{2} \sum_{j=N_{1}}^{\infty} \frac{\left|\lambda_{n}\right|}{\left|\lambda_{j}\right|-\left|\lambda_{n}\right|} \\
& \leq \frac{3}{2} \sum_{j=N_{1}}^{\infty} \frac{q^{j-n}\left|\lambda_{j}\right|}{\left|\lambda_{j}\right|-q^{j-n}\left|\lambda_{j}\right|} \\
& =\frac{3}{2} \sum_{j=N_{1}}^{\infty} \frac{q^{j-n}}{1-q^{j-n}} .
\end{aligned}
$$

Now notice that this sum converges by the ratio test, since

$$
\begin{aligned}
\limsup _{j \rightarrow \infty}\left(\frac{q^{j-n+1}}{1-q^{j-n+1}}\right)\left(\frac{1-q^{j-n}}{q^{j-n}}\right) & =\limsup _{j \rightarrow \infty}\left(\frac{q-q^{j-n+1}}{1-q^{j-n+1}}\right) \\
& =q \\
& <1 .
\end{aligned}
$$

This means $\sum_{j=N_{1}}^{\infty}\left|\log \left(\frac{\lambda_{j}}{\lambda_{j}-\lambda_{n}}\right)\right|<\infty$, and hence, $\sum_{j=N_{1}}^{\infty} \log \left(\frac{\lambda_{j}}{\lambda_{j}-\lambda_{n}}\right)$ converges. It is enough to conclude $\prod_{j=N_{1}}^{\infty} \frac{\lambda_{j}}{\lambda_{j}-\lambda_{n}}$ converges to a nonzero complex number. Therefore, $\beta_{n}$ is well-defined and nonzero for any nonnegative integer $n$.

Next, we wish to see $\lim \sup \left|\beta_{n}\right|^{\frac{1}{n}}=0$. Let us assume $n>N_{0}$ and recall equation 1 ,

$$
\begin{aligned}
\left|\beta_{n}\right| & \leq \prod_{j=0}^{N_{0}-1} \frac{\left|\lambda_{j}\right|}{\left|\lambda_{j}-\lambda_{n}\right|} \prod_{j=N_{0}}^{n-1} \frac{\left|\lambda_{j}\right|}{\left|\lambda_{n}\right|-\left|\lambda_{j}\right|} \prod_{j=n+1}^{\infty} \frac{\left|\lambda_{j}\right|}{\left|\lambda_{j}\right|-\left|\lambda_{n}\right|} \\
& \leq \prod_{j=0}^{N_{0}-1} \frac{\left|\lambda_{j}\right|}{\left|\lambda_{j}-\lambda_{n}\right|} \prod_{j=N_{0}}^{n-1} \frac{q^{n-j}\left|\lambda_{n}\right|}{\left|\lambda_{n}\right|-q^{n-j}\left|\lambda_{n}\right|} \prod_{j=n+1}^{\infty} \frac{\left|\lambda_{j}\right|}{\left|\lambda_{j}\right|-q^{j-n}\left|\lambda_{j}\right|} .
\end{aligned}
$$

MISSOURI J. OF MATH. SCI., SPRING 2016 


\section{HENTHORN-BAKER}

Therefore, for all $n \geq N_{0}$,

$$
\left|\beta_{n}\right| \leq \prod_{j=0}^{N_{0}-1} \frac{\left|\lambda_{j}\right|}{\left|\lambda_{j}-\lambda_{n}\right|} \prod_{j=N_{0}}^{n-1} \frac{q^{n-j}}{1-q^{n-j}} \prod_{j=n+1}^{\infty} \frac{1}{1-q^{j-n}} .
$$

Thus,

$$
\begin{aligned}
& \quad \limsup \left|\beta_{n}\right|^{\frac{1}{n}} \\
& \leq \limsup \left[\prod_{j=0}^{N_{0}-1} \frac{\left|\lambda_{j}\right|}{\left|\lambda_{j}-\lambda_{n}\right|}\right]^{\frac{1}{n}} \limsup \left[\prod_{j=N_{0}}^{n-1} \frac{q^{n-j}}{1-q^{n-j}}\right]^{\frac{1}{n}} \\
& \quad \times \limsup \left[\prod_{j=n+1}^{\infty} \frac{1}{1-q^{j-n}}\right]^{\frac{1}{n}} .
\end{aligned}
$$

We now wish to show

$$
\limsup \left[\prod_{j=n+1}^{\infty} \frac{1}{1-q^{j-n}}\right]^{\frac{1}{n}}=1
$$

or equivalently,

$$
\limsup _{n \rightarrow \infty} \frac{\sum_{j=n+1}^{\infty} \log \left(\frac{1}{1-q^{j-n}}\right)}{n}=\limsup _{n \rightarrow \infty} \frac{\sum_{k=1}^{\infty} \log \left(\frac{1}{1-q^{k}}\right)}{n}=0 .
$$

But this is true, since $\sum_{k=1}^{\infty} \log \left(\frac{1}{1-q^{k}}\right)$ is finite by the ratio test, as shown below:

$$
\begin{aligned}
\lim _{k \rightarrow \infty} \frac{\left|\log \left(\frac{1}{1-q^{k+1}}\right)\right|}{\left|\log \left(\frac{1}{1-q^{k}}\right)\right|} & =\lim _{k \rightarrow \infty}\left|\frac{\log \left(1-q^{k+1}\right)}{\log \left(1-q^{k}\right)}\right| \\
& =\lim _{k \rightarrow \infty}\left|\frac{\left(\frac{1}{1-q^{k+1}}\right)\left[-q^{k+1} \log (q)\right]}{\left(\frac{1}{1-q^{k}}\right)\left[-q^{k} \log (q)\right]}\right| \\
& =\lim _{k \rightarrow \infty}\left|\frac{-q\left(1-q^{k}\right)}{1-q^{k+1}}\right| \\
& =q \\
& <1 .
\end{aligned}
$$




\section{AN INFINITE SERIES APPLIES TO OPERATOR THEORY}

Next, we wish to see

$$
\limsup \left[\prod_{j=N_{0}}^{n-1} \frac{q^{n-j}}{1-q^{n-j}}\right]^{\frac{1}{n}}=0,
$$

or equivalently

$$
\limsup _{n \rightarrow \infty} \frac{\sum_{j=N_{0}}^{n-1} \log \left(\frac{q^{n-j}}{1-q^{n-j}}\right)}{n}=-\infty .
$$

But note, since $\sum_{\ell=1}^{\infty}\left|\log \left(1-q^{\ell}\right)\right|=\sum_{\ell=1}^{\infty} \log \left(\frac{1}{1-q^{\ell}}\right)$ converges, as before, we have

$$
\begin{aligned}
& \limsup \frac{\sum_{j=N_{0}}^{n-1} \log \left(\frac{q^{n-j}}{1-q^{n-j}}\right)}{n} \\
& =\limsup \frac{\log (q) \sum_{j=N_{0}}^{n-1}(n-j)-\sum_{j=N_{0}}^{n-1} \log \left(1-q^{n-j}\right)}{n} \\
& =\limsup \frac{\log (q) \sum_{j=N_{0}}^{n-1}(n-j)+\sum_{j=N_{0}}^{n-1}\left|\log \left(1-q^{n-j}\right)\right|}{n} \\
& =\limsup \frac{\log (q) \sum_{\ell=1}^{n-N_{0}} \ell+\sum_{\ell=1}^{n-N_{0}}\left|\log \left(1-q^{\ell}\right)\right|}{n} \\
& \leq \limsup \frac{\log (q) \frac{\left(n-N_{0}\right)\left(n-N_{0}-1\right)}{2}+\sum_{\ell=1}^{\infty}\left|\log \left(1-q^{\ell}\right)\right|}{n} \\
& =-\infty .
\end{aligned}
$$

Finally, notice that since $\left|\lambda_{n}\right| \longrightarrow \infty$ for each fixed $j$, we get that for each $j$ with $0 \leq j \leq N_{0}-1, \frac{\left|\lambda_{j}\right|}{\left|\lambda_{j}-\lambda_{n}\right|} \longrightarrow \infty$ as $n \longrightarrow \infty$. Therefore, for each $j$ with $0 \leq j \leq N_{0}-1$, there exists a positive integer $M_{j}$ such that for all $n \geq M_{j}$,

$$
\frac{\left|\lambda_{j}\right|}{\left|\lambda_{j}-\lambda_{n}\right|}<\frac{1}{2}
$$

Therefore, for all $n \geq M:=\max \left\{M_{j}: 0 \leq j \leq N_{0}\right\}$,

$$
\prod_{j=0}^{N_{0}-1} \frac{\left|\lambda_{j}\right|}{\left|\lambda_{j}-\lambda_{n}\right|}<\left(\frac{1}{2}\right)^{N_{0}}
$$

and hence,

$$
\limsup _{n \rightarrow \infty}\left[\prod_{j=0}^{N_{0}-1} \frac{\left|\lambda_{j}\right|}{\left|\lambda_{j}-\lambda_{n}\right|}\right]^{\frac{1}{n}} \leq 1 .
$$

MISSOURI J. OF MATH. SCI., SPRING 2016 


\section{HENTHORN-BAKER}

Thus, limsup $|\beta|^{\frac{1}{n}} \leq(1)(0)(1)=0$, which proves $\lim \sup \left|\beta_{n}\right|^{\frac{1}{n}}=0$.

Now, define $\omega_{n}:=\beta_{n} \lambda_{n}$ for all $n \geq 0$. Note that since $D$ is continuous, we know $\lim \sup \left|\lambda_{n}\right|^{1 / n}<\infty$ (see Theorem 2), and hence, $\lim \sup \left|\omega_{n}\right|^{1 / n}=0$.

We now show for all $k \geq 0$,

$$
\sum_{n=0}^{\infty} \omega_{n} \lambda_{n}^{k}=0
$$

or equivalently that for all $k \geq 0$,

$$
\sum_{n=0}^{\infty} \beta_{n} \lambda_{n}^{k+1}=0
$$

Now, if we let $m=k+1$, it is equivalent to show that for all $m \geq 1$,

$$
\sum_{n=0}^{\infty} \beta_{n} \lambda_{n}^{m}=0
$$

Fix $m \geq 1$. Recall Lemma 1, which tells us that $\sum_{n=0}^{N} \beta_{n}^{(N)} \lambda_{n}^{m}=0$ for $N \geq m$, and observe

$$
\begin{aligned}
\lim _{N \rightarrow \infty}\left|\sum_{n=0}^{N} \beta_{n} \lambda_{n}^{m}\right| & =\lim _{N \rightarrow \infty}\left|\sum_{n=0}^{N} \beta_{n} \lambda_{n}^{m}-\sum_{n=0}^{N} \beta_{n}^{(N)} \lambda_{n}^{m}\right| \\
& \leq \lim _{N \rightarrow \infty} \sum_{n=0}^{N}\left|\lambda_{n}\right|^{m}\left|\beta_{n}-\beta_{n}^{(N)}\right| \\
& =\lim _{N \rightarrow \infty} \sum_{n=0}^{\infty} f_{N}(n),
\end{aligned}
$$

where

$$
f_{N}(n):= \begin{cases}0 & \text { if } n>N \\ \left|\lambda_{n}\right|^{m}\left|\beta_{n}-\beta_{n}^{(N)}\right| & \text { if } n \leq N\end{cases}
$$

Now consider for all $n \geq N_{0}+1$,

$$
f(n):=2\left|\lambda_{n}\right|^{m} \prod_{j=0}^{N_{0}-1} \frac{\left|\lambda_{j}\right|}{\left|\lambda_{j}-\lambda_{n}\right|} \prod_{j=N_{0}}^{n-1} \frac{q^{n-j}}{1-q^{n-j}} \prod_{j=n+1}^{\infty} \frac{1}{1-q^{j-n}} .
$$

We shall see that $\left|f_{N}(n)\right| \leq f(n)$ for all $n \geq N_{0}+1$. 


\section{AN INFINITE SERIES APPLIES TO OPERATOR THEORY}

Notice that for $N>n \geq N_{0}+1$, equation 1 gives us

$$
\begin{aligned}
\left|\beta_{n}^{(N)}\right| & \leq \prod_{j=0}^{N_{0}-1} \frac{\left|\lambda_{j}\right|}{\left|\lambda_{j}-\lambda_{n}\right|} \prod_{j=N_{0}}^{n-1} \frac{\left|\lambda_{j}\right|}{\left|\lambda_{n}\right|-\left|\lambda_{j}\right|} \prod_{j=n+1}^{N} \frac{\left|\lambda_{j}\right|}{\left|\lambda_{j}\right|-\left|\lambda_{n}\right|} \\
& \leq \prod_{j=0}^{N_{0}-1} \frac{\left|\lambda_{j}\right|}{\left|\lambda_{j}-\lambda_{n}\right|} \prod_{j=N_{0}}^{n-1} \frac{\left|\lambda_{j}\right|}{\left|\lambda_{n}\right|-\left|\lambda_{j}\right|} \prod_{j=n+1}^{\infty} \frac{\left|\lambda_{j}\right|}{\left|\lambda_{j}\right|-\left|\lambda_{n}\right|} \\
& \leq \prod_{j=0}^{N_{0}-1} \frac{\left|\lambda_{j}\right|}{\left|\lambda_{j}-\lambda_{n}\right|} \prod_{j=N_{0}}^{n-1} \frac{q^{n-j}\left|\lambda_{n}\right|}{\left|\lambda_{n}\right|-q^{n-j}\left|\lambda_{n}\right|} \prod_{j=n+1}^{\infty} \frac{\left|\lambda_{j}\right|}{\left|\lambda_{j}\right|-q^{j-n}\left|\lambda_{j}\right|} \\
& =\prod_{j=0}^{N_{0}-1} \frac{\left|\lambda_{j}\right|}{\left|\lambda_{j}-\lambda_{n}\right|} \prod_{j=N_{0}}^{n-1} \frac{q^{n-j}}{1-q^{n-j}} \prod_{j=n+1}^{\infty} \frac{1}{1-q^{j-n}} .
\end{aligned}
$$

By this result and equation 4 , we conclude that for $N>n \geq N_{0}+1$, both $\beta_{n}$ and $\beta_{n}^{(N)}$ are bounded by

$$
\prod_{j=0}^{N_{0}-1} \frac{\left|\lambda_{j}\right|}{\left|\lambda_{j}-\lambda_{n}\right|} \prod_{j=N_{0}}^{n-1} \frac{q^{n-j}}{1-q^{n-j}} \prod_{j=n+1}^{\infty} \frac{1}{1-q^{j-n}} .
$$

Thus, if $N>n \geq N_{0}+1$,

$$
\left|f_{N}(n)\right| \leq\left|\lambda_{n}\right|^{m}\left(\left|\beta_{n}\right|+\left|\beta_{n}^{(N)}\right|\right) \leq f(n)
$$

Also, if $n \geq N_{0}+1$ and $n>N$,

$$
\left|f_{N}(n)\right|=0 \leq f(n) .
$$

Thus, for all $n \geq N_{0}+1$,

$$
\left|f_{N}(n)\right| \leq f(n) .
$$

Next, we need to show that $\sum_{n=0}^{\infty} f(n)$ converges. We will do so by the root test. Note that by arguments given earlier in this proof and by the continuity condition on $D$ provided by Theorem 2 , it is enough to show

$$
\limsup \left[\prod_{j=N_{0}}^{n-1} \frac{q^{n-j}}{\left|1-q^{n-j}\right|}\right]^{1 / n} \limsup \left[\prod_{j=n+1}^{\infty} \frac{1}{\left|1-q^{j-n}\right|}\right]^{1 / n}=0 .
$$

However, by prior arguments,

$$
\limsup \left[\prod_{j=N_{0}}^{n-1} \frac{q^{n-j}}{1-q^{n-j}}\right]^{1 / n} \limsup \left[\prod_{j=n+1}^{\infty} \frac{1}{1-q^{j-n}}\right]^{1 / n}=(0)(1)
$$

proving $\limsup |f(n)|^{\frac{1}{n}}=0<1$. Thus, $\sum_{n=1}^{\infty} f(n)$ converges.

Now, we can apply the Lebesgue Dominated Convergence Theorem, 


\section{HENTHORN-BAKER}

$$
\begin{aligned}
\lim _{N \rightarrow \infty} \sum_{n=0}^{\infty} f_{N}(n) & =\lim _{N \rightarrow \infty}\left[\sum_{n=0}^{N_{0}} f_{N}(n)+\sum_{n=N_{0}+1}^{\infty} f_{N}(n)\right] \\
& =\sum_{n=0}^{N_{0}} \lim _{N \rightarrow \infty} f_{N}(n)+\sum_{n=N_{0}+1}^{\infty} \lim _{N \rightarrow \infty} f_{N}(n) \\
& =0 .
\end{aligned}
$$

This proves that $\lim _{N \rightarrow \infty}\left|\sum_{n=0}^{N} \beta_{n} \lambda_{n}^{m}\right|=0$, and thus, $\sum_{n=0}^{\infty} \beta_{n} \lambda_{n}^{m}=0$ for all integers $m \geq 1$.

Now recall that no $\beta_{n}$ is zero, and no $\lambda_{n}$ is zero. Thus, $\left\{\omega_{n}\right\}$ is a sequence of well-defined complex numbers which are never zero, and hence nontrivial.

Therefore, $\left\{\omega_{n}\right\}$ satisfies condition (v) in Theorem 3 needed for an operator to fail spectral synthesis on $H(\mathbb{C})$, and we can conclude that $D: H(\mathbb{C}) \rightarrow H(\mathbb{C})$ defined as $D\left(\sum_{n=0}^{\infty} a_{n} z^{n}\right)=\sum_{n=0}^{\infty} a_{n} \lambda_{n} z^{n}$ is a nonsynthetic cyclic diagonal operator.

\section{Conclusion}

Before this particular theorem, it was not known if there existed even one operator failing spectral synthesis on the space $H(\mathbb{C})$. Now we have an infinite class of operators doing just that. For example, if $D$ is a diagonal operator with eigenvalues $\lambda_{n}:=b^{n}$ for any $b>1, D$ is nonsynthetic on $H(\mathbb{C})$. Equation 1 leads us to the general statement that a diagonal operator on $H(\mathbb{C})$ whose eigenvalues "grow exponentially" is nonsynthetic. Even without a solid background in operator theory, one can appreciate the novelty of this sequence of complex numbers that, although nontrivial, can be used to represent zero. Perhaps there are implications beyond those in the study of spectral synthesis that will make this discovery of even more relevance in the pure mathematics community.

\section{REFERENCES}

[1] L. Brown, A. Shields, and K. Zeller, On absolutely convergent exponential sums, Trans. Amer. Math. Soc., 96 (1960), 162-183.

[2] J. B. Conway, Functions of One Complex Variable, Graduate Texts in Mathematics, Springer-Verlag, New York, 1978.

[3] I. N. Deters, A connection between operator topologies, polynomial interpolation, and synthesis of diagonal operators, J. Math. Anal. Appl., 350 (2009), 354-359.

[4] I. N. Deters and S. M. Seubert, Cyclic vectors of diagonal operators on the space of functions analytic on a disk, J. Math. Anal. Appl., 334 (2007), 1209-1219.

[5] I. N. Deters and S. M. Seubert, An application of entire function theory to the synthesis of diagonal operators on the space of entire functions, Houston Journal of Mathematics, 38.1 (2012), 201-208. 


\section{AN INFINITE SERIES APPLIES TO OPERATOR THEORY}

[6] P. Enflo, On the invariant subspaces problem for Banach spaces, Acta Math., 158 (1987), 213-313.

[7] M. Henthorn, Examples of Diagonal Operators that Fail Spectral Synthesis on Spaces of Analytic Functions, a dissertation, Bowling Green State University, 2011.

[8] D. Kalman, The Generalized Vandermonde Matrix, Mathematics Magazine, 57 (1984), 15-21.

[9] J. Marin Jr. and S. M. Seubert, Cyclic vectors of diagonal operators on the space of entire functions, J. Math. Anal. Appl., 320 (2006), 599-610.

[10] N. K. Nikol'skii, Operators, Functions, and Systems: An Easy Reading, Vol. I: Mathematical surveys and monographs, Vol. 92, American Mathematical Society, Providence, RI, 2002.

[11] N. K. Nikol'skii, The present state of the spectral analysis-synthesis problem I, Fifteen Papers on Functional Analysis, Amer. Math. Soc. Transl., Providence, RI, 124 (1984), pp. 97-129.

[12] K. Overmoyer, Applications of Entire Function Theory to the Spectral Synthesis of Diagonal Operators, a dissertation, Bowling Green State University, 2011.

[13] D. Sarason, Invariant subspaces and unstarred operators algebras, Pacific J. Math., 17 (1966), 511-517.

[14] D. Sarason, Weak-star density of polynomials, J. Reine Angew. Math., 252 (1972), $1-15$.

[15] J. E. Scroggs, Invariant subspaces of normal operators, Duke Math. J., 26 (1959), 95-112.

[16] S. M. Seubert, Spectral synthesis of diagonal operators on the space of entire functions, Houston Journal of Mathematics, 34.3 (2008), 807-816.

[17] S. M. Seubert and J. Gordon Wade, Spectral synthesis of diagonal operators and representing systems on the space of entire functions, J. Math. Anal. Appl., 344 (2008), 9-16.

[18] R. V. Sibilev, Uniqueness theorem for Wolff-Denjoy series, Algebra i Analiz, 7 (1995), 170-199, English Translation in St. Petersburg Math. J., 7 (1996), 145-168.

[19] J. Wermer, On invariant subspaces and normal operators, Proc. Amer. Math. Soc., 3 (1952), 270-277.

[20] J. Wolff, Sur les series $\sum A_{k} /\left(z-z_{k}\right)$, Comptes Rendus, 173 (1921), 1057-1058, $1327-1328$.

[21] B. S. Yadav, The present state and heritages of the invariant subspace problem, Milan Journal of Mathematics, 73 (2005), 289-316.

MSC2010: 15A00, 30D00, 40A00, 40C00, 47B00

Key words and phrases: Operator theory, linear algebra, Vandermonde matrix applications, series, Cramer's Rule applications

Mathematics and Computer Science Department, Edinboro University of Pennsylvania, 219 Meadville Street, Edinboro, PA 16444

E-mail address: mbaker@edinboro.edu

MISSOURI J. OF MATH. SCI., SPRING 2016 